\title{
MODELLING THE UNSTEADY FLOW OF WATER INTO A PARTLY SATURATED SOIL
}

\begin{abstract}
Benyelles ZOHEIR - Department of Civil Engineering, Faculty of Technology,University Aboubekr BELKAID, Tlemcen, Algeria, email: z_benyelles@mail.univ-tlemcen.dz

Abdeldjalil ZADJAOUI - Department of Civil Engineering, Faculty of Technology,University Aboubekr BELKAID, Tlemcen, Algeria, email: a_zadjaoui@mail.univ-tlemcen.dz

Bekkouche ABDELMALEK - Department of Civil Engineering, Faculty of Technology,University Aboubekr BELKAID, Tlemcen, Algeria, email: z_benyelles@mail.univ-tlemcen.dz

Abstract: Flows in unsaturated medium are frequent in the field of civil engineering and more particularly in geotechnics. The study undertaken here tries to solve the unsaturated transient flow equation in porous media using the finite element method. Numerical solution of a finite element discretization is used along with an implicit integration scheme of the time stepping. A functional one that makes it possible to find the distribution of the hydraulic load has been proposed and a calculation program has been developed. The results obtained by this program called TFAP (Transient Flow Analysis Program) are compared to other previous work in the subject. The authors show the importance of this study through two real examples. Liakopoulos conducted several experiments on the water drainage through a vertical column filled with Del Monte sand. One of these experiments was chosen to perform a simulation by the model. The results of the calculation are compared with the experimental data as well.
\end{abstract}

Keywords: Flow, Unsaturated porous media, Modelling, FEM

\section{Introduction}

Solving flow problems is of vital importance since it concerns almost all science and water engineering: civil engineering for flow in dikes and earth dams and underground work, the hydrogeology for the estimation and management of water resources in aquifers; hydrology for the coupling between surface water and groundwater; agronomy for interactions between water, plants and soil, irrigation of cropland and drainage of flooded soils. The distribution of water in soils depends on the hydrogeological conditions of the site considered. In general, the porous media involves both the saturated zone, where the pores are completely filled with water, and the unsaturated zone. The saturated portion which extends to the substratum is limited upwards by the free surface, defined as the locus of points where the water pressure is equal to the atmospheric pressure. Beyond the free surface, negative pore water pressures (suction) exist, and experience shows that the further away from the free surface, the smaller the degree of saturation $\mathrm{Sr}$ (or volumetric water content $\theta$ decreases). A review of a water current profile of a solid leads to the distinction of a near-saturated area located in close proximity of the free surface called the capillary fringe. This paper presents a numerical formulation (variational principle) of transient flow in unsaturated porous media.

\section{Background, previous work and problems}

The equations of the model do not take into account the interaction of the deformations of the skeleton, it is only about the water aspect, and that is to say the flow of water and air as well as the movement of dissolved air in water. The numerical solution of these equations associates the finite element method to discretize space and an implicit integration scheme to time-stepping.

The modeling of the water behavior of unsaturated soils has inadequate consensus among geotechnical specialists. The extension of Darcy's law, as proposed by Darcy in 1956 as a framework for describing the movement of water in soils, has been the subject of various criticisms, often supported by laboratory experimental results $[1,2,3,4]$. Its generalization, like 
those of Buckingham [5], Richards [6], Childs and Collis-George [7], seems to provide a more complete answer and better describe reality through physical modeling with two variable fields.

Different theoretical and numerical models based on physical models have long existed, such as those of Philip, [8], Bruce and Klute [9], Vachaud [1], Philip [10, 11], Childs [3], and MorelSeytoux and Billica $[12,13]$.

Currently, the equations used to describe the flow of fluid through porous media are mainly based on the semi-empirical equations given by Buckingham [5], and Richards [6]. Despite limitations and disadvantages, the Richards equation is still the most widely used equation for modeling unsaturated soil flow [14] both numerically and experimentally. Due to the large and diverse applications of the problems, much research has been devoted to an adequate assessment of the various forms of the Richards equation. Both analytical and numerical approaches have been studied in the literature. However, the analytical solutions of the Richards equation are rather rare and are generally limited to particular cases [15, 16, and 17]. This is mainly due to the dependence relationship between the hydraulic conductivity and the suction storage $[15,16$, and 18]. Consequently, the uses of many numerical methods to the solution of Richards's equation with various applications in civil engineering have been identified in previous work. Finite element methods and finite difference methods have been adopted by several researchers $[19,20,12,13$, 21, 22, and 23]. Ross [24] introduced an efficient non-iterative solution for the Richards equation using soil property descriptions as proposed by Brooks and Corey [25]. In this method, Ross used a discretization scheme of space and time to derive a set of tri-diagonal linear equations that were then solved in a non-iterative manner. Varado et al. [26] later carried out an in-depth evaluation of Ross's methodology and concluded that the model provides robust and accurate solutions to available analytical ones [27].

Although different quasi-analytical approaches have been developed in the literature, they are applicable only to initial conditions and to particular limitations rarely encountered in practice.

Several other iterative solutions have also been cited in the literature, for example by Farthing and al. [28], who used the well-known pseudo-transient continuation approach to solve the nonlinear transient water infiltration problem, The authors recommended stability diagrams for the exact solution of the Richards equation. In geo-environment applications, Bunsri et al. [29] solved the Richards's equation with advection-dispersion and solute transport equations by the Galerkin technique.

Witelski [30] used perturbation methods to study the interaction of wetting fronts with impermeable boundaries in stratified soils governed by the Richards equation by comparison with numerical solutions, Witelski concluded that disturbance methods are capable of giving very precise solutions of the Richards equation [30]. In 2009 Zadjaoui [31, 32] studied the same problem in an experimental way. The results were consistent with those of Witelski's work.

Each method mentioned above makes it possible to solve Richards's equation in a different way. Frequently, hypotheses are formulated and empirical models are implemented in order to overcome difficulties in solving the equation linked to the strong interdependence of some of the parameters involved, often resulting from experimentation. Analytical solutions are part of classical perturbation methods [33, 34, and 35]. However, as with other analytical techniques, some restrictions bounds the large-scale application of disturbance methods, the most important is the interdependency of the parameters (conductivity and capillary capacity) of the desired variable, namely suction and/or the volume water content. This complicates the strong non-linearity as the parameters do not seem to follow a strict rule. In addition, the approximate solutions solved by the perturbation methods are valid, in most cases. At this level it is important to mention two powerful analytical methods, namely Homotopy Perturbation Method (HPM) [36, 37, 38, 39, 40, and 41] and the Method of Variational Iteration (VIM) [42, 43, 44, 45, and 46] which have been used to solve the problem of one-dimensional infiltration of water in unsaturated soil governed by the Richards equation [47]. HPM is a coupling of the perturbation method and the homotopy method, which eliminates the limitations of traditional disturbance methods. He proposed a VIM method 
based on the use of restricted variation and correction functional. A broad application for the solution of ordinary differential and partial nonlinear equations in general was found [42].

Shahrokhabadi et al. [48] proposed a fast-convergence solution for modeling transient flow in variably saturated soils using the isogeometric analysis.

The results show that the model performs well over a wide range of unsaturated flows while higher order finite element analysis model diverges especially in problems with high level nonlinearity. Modeling unsaturated flow through porous media is of interest in many geotechnical engineering problems. (e.g., [49, 50, 51], reinforced Earthen structures (e.g. [52]), and bearing capacity of foundations (e.g., $[53,54]$ ). Loss of suction and subsequent reduction in soil's effective stress, due to steady or transient flow can adversely impact the performance and integrity of variably saturated slopes and earthen structures (e.g., [55, 54]).

The problem is to solve a transient problem in unsaturated soil. Despite the complexity of physical phenomenon the authors have simplified the problem to a system of nonlinear equations. Lipnikova et al. [56] are proposed and analyzed a new preconditioning strategy that is based on a stable discretization of the Jacobian continuum. Unfortunately, this complex proposition does not find its place in the practice of the engineer.

\section{Basic equations}

\subsection{Equations of conservation of mass in the two media: fluid and solid}

The conservation of mass of water in an elementary volume of $\Omega \mathrm{w}$ expressed as the change in the unit of time in the mass of fluid contained in $\Omega \mathrm{w}$ is equal to the algebraic sum of the mass of fluid passing through the surface of the volume considered. This results in the following equation:

$\forall \mathrm{M} \in \Omega_{\mathrm{w}}$ we have:

$$
\operatorname{div}\left[\rho_{w}(M) v_{w}(M)\right]+\frac{\partial}{\partial t}\left[\rho_{w}(M) n S_{r}\right]+\rho_{w}(M) q_{e}=0
$$

In a similar manner the conservation of mass of the solid is:

$\forall \mathrm{M} \in \Omega_{\text {s }}$ we have:

$$
\operatorname{div}\left[\rho_{s}(M) v_{s}(M)(1-n)\right]+\frac{\partial}{\partial t}\left[\rho_{s}(M) n S_{r}\right]+\rho_{w}(M) q_{e}=0
$$

In these equations we use the following notations:

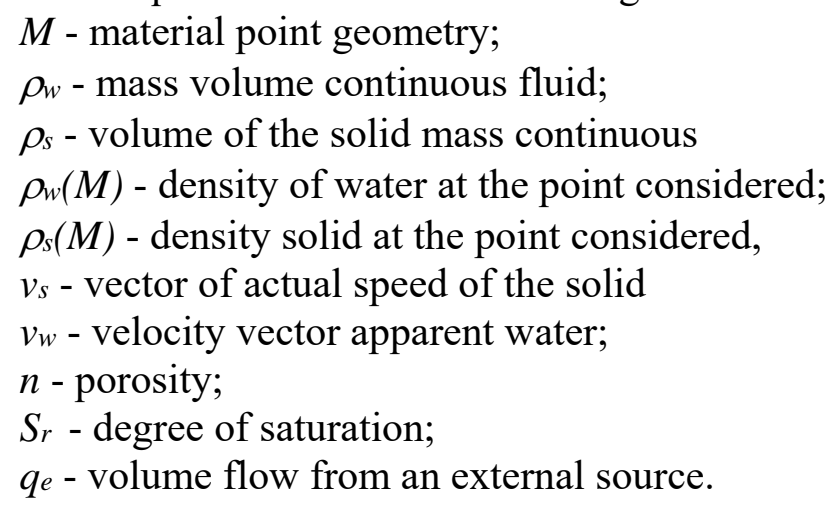

For a soil in an adiabatic state with constant density the suction does not depend on the temperature.

From this, we can say that the equation of thermodynamic state of soil can be neglected when trying to solve the flow problem in homogeneous and anisotropic soil in adiabatic state. 


\subsection{Darcy general equation}

It was verified experimentally that the flow at low Reynolds number in unsaturated soils obey the law of Darcy. It connects linearly the flow velocity and filtration in the hydraulic gradient in a linear fashion through the permeability tensor. This is the fundamental equation that translates at the macroscopic scale, the flow in a porous medium. However, it is established under certain assumptions, the main ones out below:

- The kinetic energy is negligible.

- The flow is laminar.

- The flow regime is permanent.

Subsequently, we therefore assume the validity of Darcy's law which, in its general form, is written [21, 58, and 59]:

$$
v_{w}-n S_{r} v_{s}=-k_{n s a t} \operatorname{grad}(h)
$$

Haverkamp [19] reported in the case of a partially saturated soil, that the tensor knsat may be expressed directly as a function of the water content $(\theta)$. However, the experimental determination of this relationship is difficult and imprecise especially at low water contents. To define a direct relationship between permeability and bijective and sucking, we neglect here the hysteresis phenomenon between the two processes of wetting and drainage.

We assume, moreover, that the porous medium can be studied fully characterized by two functions $s(\theta)$ and $k_{n s a t}(s)$.

The numerical point of view, as the water content undergoes a discontinuity, the use of quantities $s(\theta)$ and $k_{n s a t}(s)$ is impractical for modeling problems infiltration and evaporation in a separate domain in two areas saturated and partially saturated.

According to Humbert, [20] $\mathrm{k}_{\text {nsat }}$ tensors and $\mathrm{k}_{\text {sat }}$ are homothetic. The homothetic ratio $\mathrm{k}_{\mathrm{r}}(\mathrm{s})$ is a scalar function, its use overcomes the difficulty in question. It follows.

$$
k_{n s a t}=k_{r}(s) k_{\text {sat }}
$$

$k_{r}(s)$ is the relative permeability which reflects the effect of the non-saturation and permeability tensor $k_{\text {sat }}$.

Unlike flows in a saturated environment, which are mainly due to the total potential resulting from the gravitational potential, the matric potential which expresses the capillary forces and adsorption potential is a function of the water content. Therefore, due to the tendency of energy balance, the flow is oriented from low suctions areas to high suctions areas. Furthermore, the ratio between flow rate and water content increases with decreasing water content.

The phenomenon of unsaturated flow can occur under a variety of complex conditions and it is important to outline the particular situation to which this work applies. For this research, the behavior of the air pressure is atmospheric everywhere at all times. The soil domain in which flow takes place is homogeneous, anisotropic and non-deformable. The water is incompressible and the flow regime is laminar. Temperature changes may in fact influence the behavior of unsaturated flow since the water can be transferred as vapor as well as liquid. It is assumed in this work that isothermal conditions exist. The hysteretic form of some of the soil parameters is not included in this paper. Those were mainly the limitations and assumptions of this contribution.

\subsection{Coupling of the basic equations}

If we introduce the concept of a field derived particulate from the backbone, assuming $\rho w$ and $\rho s$ are invariable in space and by using the Eq. (1), (2) and (3) we find the following equation:

$$
\operatorname{div}\left(k_{r}(s) k_{s a t} \operatorname{grad}(h)\right)=\left(c_{w}+c_{f}+c_{p}\right) \frac{d s}{d t}+q_{e}
$$


equality in which :

$$
\begin{array}{lrl}
c_{w}=n S_{r} \beta_{w} & c_{f}=n S_{r} \beta_{f} & c_{p}=n S_{r} \beta_{p} \\
\beta_{w}=\frac{1}{\rho_{w}} \frac{d \rho_{w}}{d s} & \text { (Coefficient of water compressibility) } \\
\beta_{f}=\frac{1}{\rho_{s}} \frac{d \rho_{s}}{d s} & \text { (Coefficient of backbone compressibility) } \\
\beta_{p}=\frac{1}{S_{r}} \frac{d S_{r}}{d s}+\left[\frac{1}{n}+\frac{1}{n-1}\right] \frac{d n}{d s} & \text { (Coefficient of porous media compressibility) }
\end{array}
$$

We have $s=\gamma_{w}(h-z)$, then Eq. (5) becomes:

$$
\operatorname{div}\left[k_{r}(s) k_{\text {sat }} \operatorname{grad}(h)\right]=S_{s} \frac{d h}{d t}+q_{e}
$$

$S_{s}=\gamma_{w}\left(c_{w}+c_{f}+c_{p}\right)$ is called the coefficient of specific storage. It is equal to $\rho_{d} C(s)$

For suction based method, the governing differential equation for moisture movements in soils is defined in terms of suction by Richard's Equation:

$$
\frac{\partial}{\partial x}\left(k(s) \frac{\partial s}{\partial x}\right)+\frac{\partial}{\partial y}\left(k(s)\left(\frac{\partial s}{\partial y}+1\right)\right)=\rho_{d} \cdot C(s) \frac{\partial s}{\partial t}+q_{e}
$$

Where $k(s)$ is the coefficient of hydraulic conductivity for unsaturated soils, which is a function of matrix suction $s, C(s)$ is the specific water capacity, which is also the slope of soil water characteristic curve $\mathrm{dw} / \mathrm{ds}, \rho_{\mathrm{d}}$ is the dry density of the soil and $\mathrm{w}$ is the water content.

Eq. (7), often known as the Richards equation, has been used in geotechnical engineering and governs the transient flow of water in saturated and unsaturated porous medium.

\section{Digital presentation of the Richards equation}

Richards Eq. (7) is valid in the field $\Omega$ It is associated with two types of boundary conditions on the contour A:

A Dirichlet boundary conditions which impose the restriction that the potential is some value at some location: $h=\bar{h}(P) \quad$ on $\mathrm{A}_{\mathrm{h}} \in \mathrm{A}$;

A flow condition or requirement imposed or Newman's condition:

$$
-k_{i j} \cdot \frac{\partial h}{\partial x_{j}} n_{i}=\bar{q}(P) \quad \text { on } A_{q} \in A^{0}
$$

With $\bar{h}(P)$ : imposed potential at point $\mathrm{P}$ on the edge portion $\mathrm{A}_{\mathrm{h}}$;

$\mathrm{n}_{\mathrm{i}}$ : components of the normal vector and $\bar{q}(P)$ : imposed flux at point $\mathrm{P}$ on the edge portion $\mathrm{A}_{\mathrm{q}}$

The approach to reach the formulation finite element is a two-step process: we first reduce the problem of solving a differential system (Eq. (7)) by minimizing the functional (variational principle)

Then to solve the problem numerically, we seek an approximate solution. In finite elements this solution is defined by the data:

1. of a mesh of the computational domain $\Omega$

2. of an interpolation on each element of the mesh.

\subsection{Variational formulation on the hydraulic aspects}

Based on the different functional used for steady flow in saturated media and by analogy to problems of heat transfer, it appears that we are able to offer a functional which makes finding the 
distribution of hydraulic head. Obtained at the elementary level language that can be written as a first step in the form:

$$
\begin{aligned}
& d \Pi=\int_{\Omega}\left\{\left[k_{r}(s) k_{i j} h_{, j}\right]+\left[n \beta_{w} \gamma_{w}\left(S_{r}(s)-\frac{d s_{r}(s)}{d s}\right)-\gamma_{w} \frac{S_{r}(s)}{1-n} \frac{d n}{d s}\right] \frac{d h}{d t}\right\} d h . d \Omega- \\
& \int_{A}\left\{k_{r}(s) k_{i j} h_{, j} n_{i}\right\} \delta h d A=0
\end{aligned}
$$

This equation can be written as:

$$
\begin{aligned}
& d \Pi=\int_{\Omega}\left[k_{r}(s) k_{i j} h{ }_{, j}\right] \partial h_{i} d \Omega+\int_{\Omega} n \beta_{w} \gamma_{w} S_{r}(s) \frac{\partial h}{\partial t} \delta h . d \Omega-\int_{\Omega} n \gamma_{w} \frac{\partial S_{r}(s)}{\partial s} \frac{\partial h}{\partial t} \delta h . d \Omega- \\
& \int_{\Omega} \gamma_{w} \frac{S_{r}(s)}{1-n} \frac{d n}{d s} \frac{\partial h}{\partial t} \delta h . d \Omega+\int_{\Omega} q_{e} \delta h d A
\end{aligned}
$$

The physical significance of five terms of this expression is given below:

Physical significance of five terms of Eq. (9)

\begin{tabular}{|l|l|l|l|l|}
\hline \multicolumn{1}{|c|}{ Term 1 } & \multicolumn{1}{|c|}{ Term 2 } & \multicolumn{1}{c|}{ Term 3 } & \multicolumn{1}{c|}{ Term 4 } & \multicolumn{1}{c|}{ Term 5 } \\
\hline $\begin{array}{l}\text { Volume of water out } \\
\text { of the element } \\
\text { of water in the } \\
\text { element due to the } \\
\text { compressibility of } \\
\text { fluid }\end{array}$ & $\begin{array}{l}\text { Varying the volume } \\
\text { of water pores } \\
\text { desaturation }\end{array}$ & $\begin{array}{l}\text { Variation in water } \\
\text { volume due to } \\
\text { deformation of the } \\
\text { voids }\end{array}$ & $\begin{array}{l}\text { Volume of water } \\
\text { entering the element }\end{array}$ \\
\hline Flow rates & \multicolumn{2}{|c|}{ Source of water storage } & rates imposed \\
\hline internal exchanges & \multicolumn{2}{|c|}{ external inputs } \\
\hline
\end{tabular}

Equality (8) satisfies both boundary conditions and initial conditions. The term $\partial \mathrm{h} / \partial \mathrm{t}$ should be considered as fixed for the variation.

The finite element approximation is obtained by:

$$
h^{e}(x, y, z, t)=N(x, y, z) H^{e}(t)
$$

Where $H_{i}^{e}(t)$ is the hydraulic head of the node $\mathrm{i}$ of the element (e) and $N_{i}^{e}(x, y, z)$ is the interpolation function of node $i$ of the element $(e)$.

If we know the values of $\mathrm{H}$ at the nodes of the element then the function $\mathrm{h}$ can be uniquely defined across the entire domain.

Differentiating these equations thereafter, we obtain a system of equations that can be put in the following matrix form:

$$
-K_{E}(S) H_{E}(t) \delta H_{E}-M_{E}\left(S_{r}\right) \dot{H}_{E}(t) \delta H_{E}-Q_{E}(t) \delta H_{E}=0
$$

Where

$$
\begin{gathered}
\underbrace{K_{E}(S)=\int_{\Omega} k_{r}\left(S_{r}\right) N_{i}^{T} k_{i j} N_{, i} d \Omega}_{\text {elementary conductivity Matrix }} \\
\underbrace{M_{E}\left(S_{r}\right)=\int_{\Omega} \gamma_{w} P_{E}\left[-\beta_{w} S_{r E}(S)+C_{r E}(S)\right] N^{T} N d \Omega}_{\text {storage hydraulic matrix }} \\
\underbrace{Q^{E}(t)=\int_{A} q_{e} N d A}_{\text {hydraulic intake external vector }}
\end{gathered}
$$

With: $k_{r E}\left(S_{r}\right)$ : vector of the present values of the relative permeability at points of integration.

$S_{r E}(S)$ : a vector of the present values of the degree of saturation at the point of integration.

$P_{E}$ : vector of present values of porosity integration points.

$C_{r E}(s)$ : vector of present values of the derivative at the points of integration and $N, i$ : designating the derivative matrix of interpolation functions. 
After assembling the Eq. (10) allows to write the global matrix system as:

$$
K_{G}(h) H(t)+M_{G}(h) \dot{H}_{G}+Q_{G}(h, t)=0
$$

Where $K_{G}$ is the matrix overall flow, $M_{G}$ the total matrix storage hydraulic and $Q_{G}$ global hydraulic intake external vector.

\subsection{Integration problem in time}

In this approach, we are interested in using the sum general integration by introducing a coefficient $\alpha$, which allows depending on the value (data file) an implicit scheme $(\alpha=1)$ where semi-implicit ( $\alpha=0.67)$. This allows writing the Euler formula in the time interval $\left[\mathrm{t}_{\mathrm{i}}, \mathrm{t}_{\mathrm{i}+1}\right]$ as:

$$
\mathrm{H}=(1-\alpha) \mathrm{H}_{\mathrm{i}}+\alpha \mathrm{H}_{\mathrm{i}+1}
$$

the vector of external inputs is approached in a manner similar to the hydraulic head, either:

$$
\mathrm{Q}=(1-\alpha) \mathrm{Q}_{\mathrm{i}}+\alpha \mathrm{Q}_{\mathrm{i}+1}
$$

We finally obtain the expression of Euler

$$
\left[M+\alpha \cdot K_{i+1} \Delta t\right] H_{i+1}=\left[M-(1-\alpha) \cdot K_{i} \Delta t\right] H_{i}-\left[(1-\alpha) \cdot Q_{i}+\alpha \cdot Q_{i}\right] \Delta t
$$

We find the Euler method when $\alpha=0$ and when $\alpha=1 / 2$ we obtain the widely used Crank-Nicholson algorithm.

\section{Calculation program TFAP}

A Transient Flow Analysis Program, baptized TFAP was developed in the Department of Civil Engineering, Faculty of Engineering Sciences, University of Tlemcen (Algeria).

The program allows the modelling of a large number of civil engineering problems. It consists of four units and is a code of finite element analysis.

However, the program is better suited for the analysis of flow problems in porous media. This particular program allows describing the flows in the whole massif, while considering the continuity between unsaturated and saturated zones. It allows, moreover, studying both the evolution of the flow characteristics in transient time and the resolution of the current problem. The theoretical formulation is based on the approach of monophasic Richards, 1931, which generalizes the equation of flow to both areas of positive and negative water pressure. The discretization is an isoparametric four-node quadrilateral element.

\subsection{Impoundment dam}

\subsubsection{Overview of Testing}

A first test was performed on the filling of a dam built on compressible clay soils. The calculation used to evaluate changes in hydraulic head at the interface AB Figure 01.

Table 2 presents all the data entered into the calculation already used by Ould Amy and Magnan [57]. The initial state corresponds to the hydrostatic state in the foundation. The origin of the ascending axis consists of bedrock. It is assumed that the filling is done instantly.

The lower limit of the dam foundation is an impermeable; therefore a zero flow condition is considered. A hydraulic load is imposed to the two limits on the left and on the right. On the other hand, upstream of the barrage, as the filling takes place instantaneously, the hypothesis of imposing load is acceptable. No conditions at the AB interface are taken.

The numerical characteristics of mesh are 44 nodes and 30 elements. Each element is of quadrilateral type with 4 nodes. 


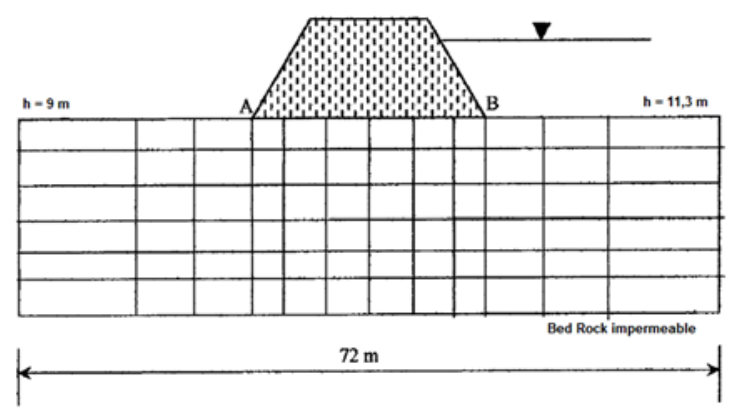

Fig 1 - AB interface between the ground and the dam with mesh and conditions to the limits

Table 2

Characteristics of the clay

\begin{tabular}{lcr}
\hline Characteristics & Units & \\
\hline Specific gravity of the water $\gamma_{\mathrm{w}}$ & $\mathrm{kN} / \mathrm{m}^{3}$ & 10 \\
\hline Porosity $\mathrm{n}$ & & 0,4 \\
\hline Compressibility of water $\beta_{\mathrm{w}}$ & $\mathrm{kPa}^{-1}$ & $10^{-3}$ \\
\hline Hydraulic Conductivity & $\mathrm{m} / \mathrm{day}$ & $10^{-4}$ \\
\hline Volumetric water content $\theta_{\text {sat }}$ & & 0,4 \\
\hline parameter ................ & $10^{-3}$ \\
\hline parameter ................. & 0,4 \\
\hline parameter .............. & $10^{-3}$ \\
\hline
\end{tabular}

\subsubsection{Analysis of results}

We study the evolution of the hydraulic load on the interface AB. In order to compare the results, we draw on the same figure the hydraulic head at different times: $t=1$ month, $t=6$ months, $t=1$ year and $t=10 y e a r s$ (Figure 2 ).
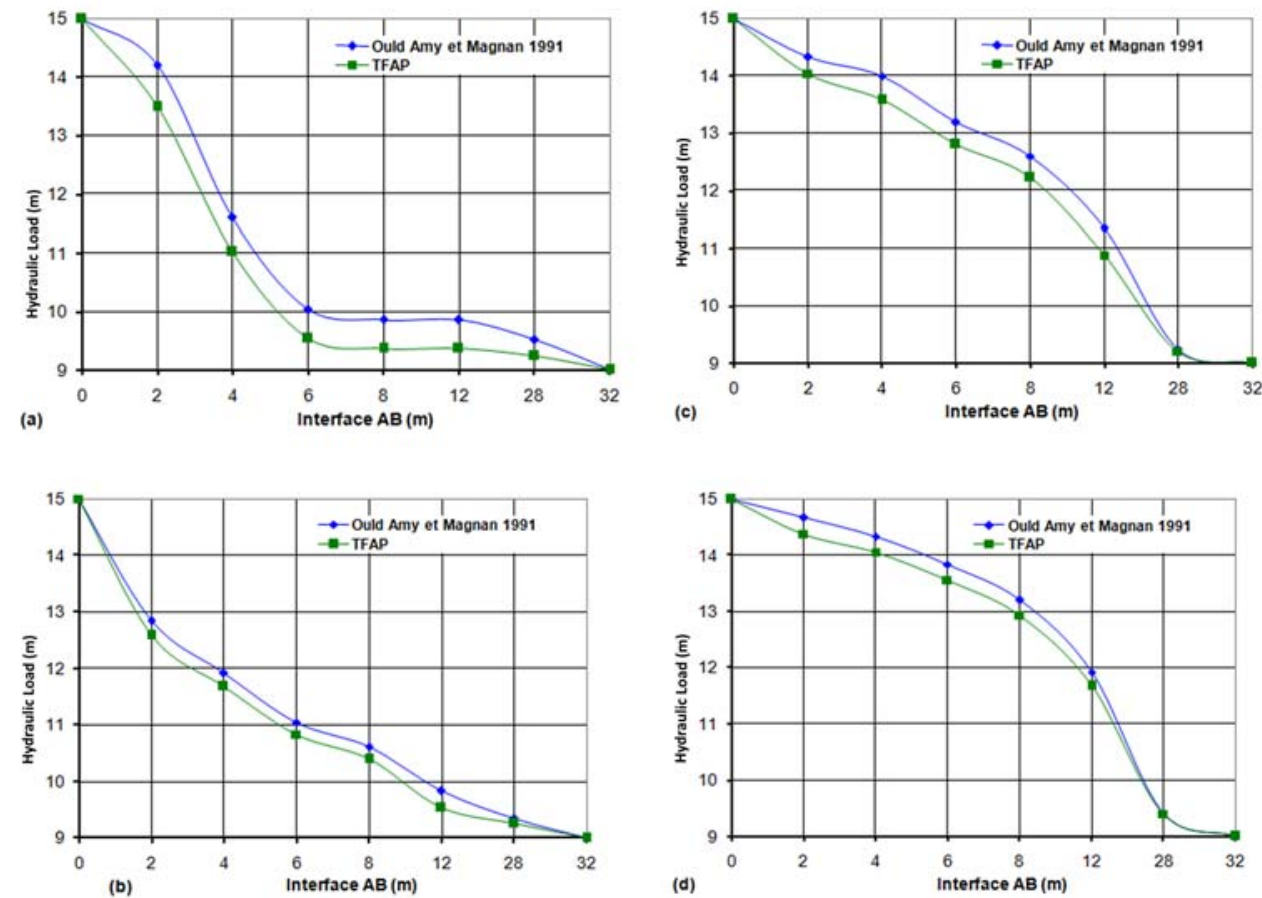

Fig 2 - Evolution of the hydraulic load on the interface $A B$ $a-t=1$ month, $b-t=6$ months, $c-t=1$ year, $d-t=10$ years

This test numerically quite severe (flooding instantaneous) relatively well converged. When the dam is impermeable, the convergence is achieved in four iterations, the foundation is completely saturated. The boundary conditions automatically cause the gradual establishment of a transient flow. The first moments, the results are quite comparable. Six months after impoundment, the difference between hydraulic loads increases significantly, especially in the upstream interface. 
The conclusion of this test is that the seal inhibits the infiltration of water inside the dam and produces a pressure drop at the interface. Further studies may be undertaken to analyze the hydrodynamic coupling to analyze the effect of the consolidation on this type of problem.

\subsection{Unsaturated soil column}

\subsubsection{Presentation of the test}

Liakopoulos [58] and Thomas [59] carried out several experiments on the drainage of water in vertical columns filled with sand from Del Monte. These experiments have been used for many comparisons of computational models. We have modeled one of them in order to contribute to the validation of our model of calculation and its programming in the program of calculation in finite elements TFAP.

The experiment was carried out on a sand column of Del Monte, one meter high $(H=1 \mathrm{~m})$. Tensiometers have been installed to measure water pressure at different levels. The experiment was carried out in several steps: for $t<0$, water was continuously poured at the top of the column, allowing it to escape freely at the bottom of the column until a steady state is established on the height of the column. At time $t=0$, the top water supply was stopped but water could still flow freely to the bottom of the column. Liakopoulos then measured the capillary tension at different times by means of tensiometers.

This test has the advantage of its simplicity and there is both experimental and numerical result.

This is a soil column height $1 \mathrm{~m}$ and $0.20 \mathrm{~m}$ wide. Hydraulic conductivity of the soil is derived from the semi-empirical equation as follows [60]:

$$
k_{n s a t}=0.106 \frac{310^{22}}{310^{22}+\left|u_{w}\right|^{18.25}}
$$

The volumetric water content directly affects the hydraulic storage coefficient. In this case the compressibility of water is assumed to be zero in order to obtain a formulation similar to Benyelles [60], ie only the storage due to the desaturation is taken into account. In this case, we have:

$$
\theta\left(u_{w}\right)=0.357 \frac{1210^{6}}{1210^{6}+\left|u_{w}\right|^{5.82}}+0.02
$$

and

$$
n \frac{\partial s_{r}}{\partial u_{w}}=0.357 \frac{5.821210^{6}}{\left(1210^{6}+\left|u_{w}\right|^{5.82}\right)^{2}}
$$

\subsubsection{Initial conditions and boundary conditions}

The initial state corresponds to the saturated state $\left(S_{r}=1\right)$ and to the hydrostatic equilibrium of the water in the column. The water pressures in the column are given by the following relations: $\gamma w(H-z)$, the pressure of air equals that of atmospheric pressure. With $\mathrm{H}$ the height of the column. For $t>0$, the bottom of the column is in contact with a reservoir of water at atmospheric pressure. As a result, at the base of the column (for $z=0$ ), the water pressure is zero. It is also considered that the flow of water entering the top of the column is zero. On the other hand, the flow of water leaving at the base of the column is free.

\subsubsection{Results and Comparisons}

Figure 3 shows the changes over time of pore pressure on the height of the column. This figure contains the results of TFAP, Liakopoulos [58], Thomas [59], Narasimhan [61] and Benyelles [60]. The short-term evolution ( 5 minutes and $10 \mathrm{~min}$ ) pressure with depth clearly shows differences between the four results due to numerical instability of the solution during the first minute of the flow and some usual experimental reading imperfections. It is difficult to achieve, in this case, similarity of the results for large depths $(>0.40 \mathrm{~m})$. 
The comparison of numerical results of Benyelles [60] and TFAP and experimental results [58, $59 ; 60]$ shows a good consistency.

It is found that the numerical model adequately describes the observed evolution of suction over time. Indeed, the difference between the experimental curves and the calculated curves remains small, even if a greater difference is noted at $t=20 \mathrm{~min}$. These curves show that the suction continues to decrease over time, reflecting the phenomenon of emptying at the bottom of the column. This phenomenon is somewhat accelerated at the beginning of the experiment but slowly slows down over time to end up in the column at a water pressure in hydrostatic equilibrium.

This may be explained by the fact that the sand contains enough fines for the column to remain saturated by capillarity, with a negative water pressure.

It is important to say at this level that the difference between the results of Benyelles [59] and TFAP (this study) can only be due to the use of different laws of conductivity and water content.
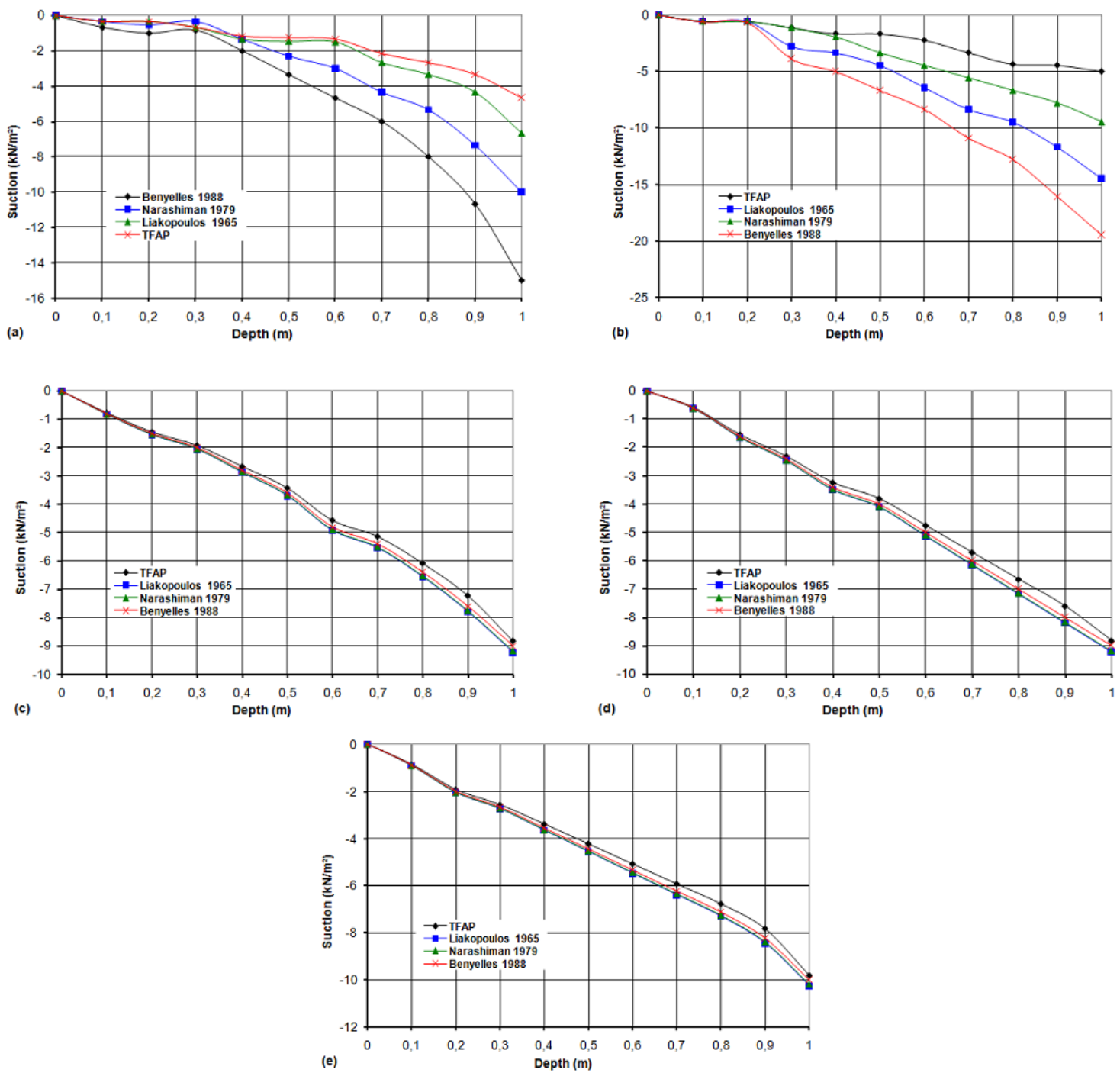

Fig 3 - Comparison of numerical and experimental results. $\mathrm{a}-\mathrm{t}=5 \mathrm{~min}, \mathrm{~b}-\mathrm{t}=10 \mathrm{~min}, \mathrm{c}-\mathrm{t}=20 \mathrm{~min} ; \mathrm{d}-\mathrm{t}=60 \mathrm{~min}$, and $\mathrm{e}-\mathrm{t}=120 \mathrm{~min}$

\section{Conclusion}

Despite simplifying assumptions, the description of unsaturated flow processes is in general very complex, because they often give rise to variations in soil moisture during flow. These variations bring about complex relationships between water content, suction and hydraulic conductivity. 
This article has summarized the equations that have been used to develop a numerical model for the analysis of unsaturated-water behavior. This model has been implemented in a finite element calculation code.

The tests carried out in one and two-dimensional cases and on two different geometries showed the aptitude of this model to satisfactorily reproduce the conditions of the tests close to the state of these materials in situ. Its developments involve the improvement of the elementary equations, in order to ensure a good representativeness of the results of the calculations. The strength of this proposal lies in its simplicity and consequently its usefulness in the practice of the geotechnical engineer.

The unsaturation of the soil is directly linked to the parameter "knsat", which itself depends on the volumetric water content and suction, this parameter only conditions the water content in the case of steady-state flows. On the other hand, in the case of a transitional regime, the change over time is conditioned by the storage coefficient (consolidation coefficient of the consolidation theory).

For the first test, it was found that the impoundment, operation and draining of the dam are strongly influenced by the evolution of the hydraulic load and therefore of the pore pressure. A damping of the flow was recorded at the interface $\mathrm{AB}$ especially as the steady state is reached.

For the column, we first recorded a very good coherence of the results with the previous work and especially the effect of the desaturation on the evolution in time of the water parameters.

In the examples, a hydraulic gradient and flow calculation is intended to ensure safety against piping and, above all, draining at the right time

The applications presented here allowed us to appreciate the degree of validity of our program. Finally, the engineer must be able to estimate all the consequences of the evolution of the hydraulic load within the infrastructure especially when a new flow regime is not reached.

\section{References}

[1] Vachaud G. (1968). Contribution à l'étude des problèmes d'écoulement en milieux poreux non saturés. PhD Thesis, Faculty of Sciences, University of Grenoble, 159 p.

[2] Ed Diny S. (1993). Etude expérimentale des transferts hydriques et du comportement mécanique d'un limon non saturé. Thesis of the INPL-National Polytechnic Institute of Lorraine. Nancy, 177p.

[3] Childs, E. C. (1969). An introduction to the physical basis of soil water phenomena. New York-London: J. Wiley and Sons. Inc. https://DOI.org/10.1180/claymin.1969.008.2.13

[4] Olsen Harold W. (1966) Darcy's law in saturated kaolinite. Water Resources Research. 2 (2).p. $287-295$. https://DOI.org/10.1029/WR002i002p00287

[5] Buckingham E. (1931). Studies on the movement of soil moisture. Bulletin 38. USDA Bureau of Soils. 30 Washington. DC. 1907.

[6] Richards L.A. Capillary conduction of $\mathrm{s}$ through porous medium. Physics 1, 318-333 https://DOI.org/10.1063/1.1745010

[7] Childs, E.C. and Collis-George G.N. (1950). The permeability of porous materials. Proceeding of the Royal Society of London Series A 201. 392-405. https://DOI.org/10.1098/rspa.1950.0068

[8] Philip J. R. (1955). The concept of diffusion applied to soil water. Proc. Nat. Acad. Sci. India 24A, pp.93-104. https://Doi.org/10.1016/0016-7061(74)90021-4

[9] Bruce R.R. and Klute A. (1956). The measurement of soil water diffusivity. Soil Science Society of American Proceeding. Vol. 20, p.458-462. Doi:10.2136/sssaj1956.03615995002000040004x

[10] Philip J. R. (1957). The theory of infiltration: 1. the infiltration equation and its solution. Soil Sci., Vol. 83, pp.345-357. DOI: 10.1097/00010694-200606001-00009

[11] Philip J. R. (1957). The theory of infiltration: 2. the profile infinity. Soil Sci., Vol. 83, pp.435-448. 1957 b.

[12] Morel-Seytoux, H.J., Billica, J.A. (1985). A Two-Phase Numerical Model for Prediction of Infiltration: Applications to a Semi-Infinite Soil Column. Water Resources Research. 21(4), p. 607-615. 1985a https://DOI.org/10.1029/WR021i004p00607

[13] Morel-Seytoux, H.J., Billica, J.A. (1985). A Two-Phase Numerical model for prediction of infiltration: case of an impervious bottom. Water Resources Research, 21(9), p. 1389-1396. 1985 b. https://DOI.org/10.1029/WR021i009p01389

[14] Hoffmann M. R. (2003). Macroscopic equations for flow in unsaturated porous media. Ph.D. dissertation, Washington University. 2003. 
[15] Ju S. H. and Kung K. J. S. (1997). Mass types, element orders and solution schemes for Richards'equation. Computers and Geosciences, 23(2), 175-187.https://DOI.org/10.1016/S0098-3004(97)85440-4

[16] Arampatzis G., Tzimopoulos C., Sakellariou-Makrantonaki M. and Yannopoulos S. (2001). Estimation of unsaturated flow in layered soils with the finite control volume method. Irrig. Drain, 50, pp. 349-358, 2001. 10.1002/ird.31

[17] Nasseri M. Shaghaghian MR., Daneshbod Y. (2008). An analytic solution of water transport in unsaturated porous media. Journal of Porous Media. 11(6). DOI: 10.1615/JPorMedia.v11.i6.60

[18] Kavetski D., Binning P. and Sloan S. W. (2002). Non-iterative time stepping schemes with adaptive truncation error control for the solution of Richards' equation. Adv. Wat. Res. 38(10), 1211-1220. https://DOI.org/10.1029/2001 WR000720

[19] Haverkamp R. (1983). Solving the equation for the infiltration of water into the soil. Analytical and numerical approaches. PhD thesis ESE-Physical Sciences: Univ. Scientific and Medical and National Polytechnic Institute of Grenoble, 240 pages.

[20] Humbert P. (1984). Application of finite element method for flow in porous media. LPC newsletter, No. 132 , p. 21-37. 1984.

[21] Baca R. G., Chung J. N. and Mulla D. J. (1997). Mixed transform finite element method for solving the nonlinear equation for flow in variably saturated porous media. Int. J. Numer. Method. Fluid. 24, 441455.https://DOI.org/10.1002/(SICI)1097-0363(19970315)24:5<441::AID-FLD501>3.0.CO;2-9

[22] Bergamaschi L. and Putti, M. (1999). Mixed finite element and Newton-type linearizations for the solution of Richards' equation. Int. J. Numer. Method. Eng., 45, 1025-1046. https://DOI.org/10.1002/(SICI) 10970207(19990720) 45:8\%3C1025::AID-NME615\%3E3.0.CO;2-G

[23] Kormi T. (2003). Modélisation numérique du gonflement des argiles non saturées. PhD Thesis, National School of Bridges and Roads, Paris, 153 pages. 2003.

[24] Ross PJ. (2003). Modeling soil water and solute transport - fast, simplified numerical solutions. Agr. J., 95, 1352-1361. DOI: 10.2134/agronj2003.1352

[25] Brooks R.H., Corey A.T. (1964). Hydraulic properties of porous media. Hydrology Paper, 3, Colorado State University, Fort Collins, 24 p.

[26] Varado N., Braud I., Ross P. J. and Haverkamp R. (2006). Assessment of an efficient numerical solution of the 1D Richards equation on bare soil. J. Hydrol., 323, 244-257. DOI: 10.1016/j.jhydrol.2005.07.052

[27] Basha, H. A. (1999). Multidimensional linearized nonsteady infiltration with prescribed boundary conditions at the soil surface. Water Resour. Res., 25(1), 75-93. https://DOI.org/10.1029/1998WR900015

[28] Farthing M. W., Kees C. E., Coffey T. S., Kelley C. T. and Miller C. T. (2003). Efficient steady state solution techniques for variably saturated groundwater flow. Adv. Water Resour., 26(8), 15 833-849. DOI:10.1016/S0309-1708(03)00076-9.

[29] Bunsri T., Sivakumar M., and Hagare D. (2008). Numerical modeling of tracer transport in unsaturated porous media. J. Appl. Fluid Mech., 1(1), 62-70. https://DOI.org/10.1007/s11242-013-0138-x

[30] Witelski T.P. (1997). Perturbation analysis for wetting front in Richards'equation. Transport Porous Med. 27, pp. 121-134. DOI: 10.1023/A:1006513009125

[31] Zadjaoui A. (2009). Etude du transfert hydrique dans les sols non saturés: échange sol - atmosphère. PhD Thesis. Department of Civil Engineering, University of Tlemcen. Algeria.

[32] Zadjaoui A. and Houmadi Y. (2019). Theoretical and experimental aspects of flow tests in situ, Algérie équipement, 28 (60), 61-69.

[33] Kevorkian J. and Cole J. D. (1996). Multiple Scale and Singular Perturbation Methods. Springer-Verlag. New York. DOI: 10.1007/978-1-4612-3968-0

[34] Nayfeh A. H. (1973). Perturbation Methods. John Wiley \& Sons, New York. 1973. DOI:10.1002/9783527617609

[35] Nayfeh A. H. and Mook D. T. (1979). Nonlinear Oscillations. John Wiley \& Sons, New York. DOI:10.1002/9783527617586

[36] He, J.H. Homotopy perturbation technique. Comput. Meth. Applied Mech. Eng., 178 (3-4): 257 -262. https://DOI.org/10.1016/S0045-7825(99)00018-3

[37] He, J.H. (2006). New interpretation of homotopy perturbation method. Int. J. Mod. Phys. B., 20 (18): $2561-2568$. https://DOI.org/10.1142/S0217979206034819

[38] Barari A., Omidvar M., Ghotbi A. R. and Ganji D. D. (2008). Application of homotopy perturbation method and variational iteration method to nonlinear oscillator differential equations. Acta Appl. Math. 104, 161-171. https://DOI.org/10.1007/s10440-008-9248-9

[39] Barari A., Ghotbi A. R., Farrokhzad F. and Ganji D. D.(2008). Variational iteration method and Homotopyperturbation method for solving different types of wave equations. J. Appl. Sci., 8, 15 120-126. 2008b. DOI: 10.3923/jas.2008.120.126

[40] Ghotbi A. R., Avaei A., Barari A. and Mohammadzade M. A. (2008). Assessment of He's homotopy perturbation method in Burgers and coupled Burgers' equations. J. Appl. Sci., 8, 322-327. 2008 a. DOI: 10.3923 /jas.2008.322.327

[41] Ghotbi A. R., Barari A., and Ganji D. D. (2008). Solving ratio-dependent predator-prey system with constant effort harvesting using homotopy perturbation method. J. Math. Prob. Eng. http://dx.DOI.org/10.1155/2008/945420 
[42] He, J.H. (1999). Variational iteration method: A kind of nonlinear analytical technique: Sorne examples. Int. Non!. Mech., 344: 699-708. DOI:10.1016/S0020-7462(98)00048-1

[43] He, J.H. (2000). Variational iteration rnethod for autonomous ordinary differential systems. Applied Math. Comput., 114 (2-3): 115-123. https://DOI.org/10.1016/S0096-3003(99)00104-6

[44] Momani S. and Abuasad S. (2006). Application of He's variational iteration method to Helmholtz equation. Chaos Soliton Fract. 27. p. 1119-1123. DOI: 10.1016/j.chaos.2005.04.113

[45] Sweilam N. H. and Khader M. M. (2007). Variational iteration method for one dimensional nonlinear thermoelasticity. Chaos Soliton Fract., 32, 145-149. http://dx.DOI.org/10.1016/j.chaos.2005.11.028

[46] Barari A., Omidvar M., Ganji D. D. and Tahmasebi Poor A. (2008). An Approximate solution for boundary value problems in structural engineering and fluid mechanics. J. Math. Problems Eng. 1-13. http://dx.DOI.org/10.1155/2008/394103

[47] Asgari A., Bagheripourb M.H., Mollazadehb M. (2011). A generalized analytical solution for a nonlinear infiltration equation using the exp-function method. Scientia Iranica, Transactions A: Civil Engineering; 18 pp. 28-35. https://DOI.org/10.1016/j.scient.2011.03.004

[48] Shahrokhabadi S., Vahedifard F. and Bhatia M. (2017). A Fast-Convergence Solution for Modeling Transient Flow in Variably Saturated Soils Using the Isogeometric Analysis. Geotechnical Frontiers. GSP 280. Pp. 756765. DOI: $10.1061 / 9780784480472.080$

[49] Lu, N., and Godt, J. W. (2013). Hillslope hydrology and stability. Cambridge University Press. https://DOI.org/10.1017/CBO9781139108164.001

[50] Griffiths, D. V., Lu, N. (2005). Unsaturated slope stability analysis with steady infiltration or evaporation using elasto-plastic finite elements. Int. J. Num. Anal. Methods. Geomech, 29(3), 249-267. https://DOI.org/10.1002/nag.413

[51] Vahedifard F., Leshchinsky D., Mortezaei K., Lu N. (2016). Effective stress-based limit-equilibrium analysis for homogeneous unsaturated slopes. Inter. J. Geom.16 (6). D4016003. DOI/10.1061/(ASCE)GM.19435622.0000554

[52] Vahedifard F., Mortezaei K., Leshchinsky B.A. (2016). Role of suction stress on service state behavior of geosynthetic-reinforced soil structures. Trans. Geotechnics, 8. p. 45-56. DOI: 10.1016/j.trgeo.2016.02.002

[53] Vanapalli S.K., Mohamed F.M.O. (2007). Bearing capacity of model footings in unsaturated soils. Experimental unsaturated soil mechanics, 483-493. 2007. https://DOI.org/10.1007/3-540-69873-6_48

[54] Vahedifard F, Robinson JD. (2015). Unified method for estimating the ultimate bearing capacity of shallow foundations in variably saturated soils under steady flow. Journal of Geotechnical and Geoenvironmental Engineering 142 (4). p.04015095. DOI: 10.1061/(ASCE)GT.1943-5606.0001445

[55] Vahedifard F., Leshchinsky BA., Mortezaei K., Lu N. (2015). Active earth pressures for unsaturated retaining structures. J. Geot. and Geoe. Eng. 141 (11). p.04015048. DOI: 10.1061/(ASCE)GT.1943-5606.0001356

[56] Lipnikova K., Moultona D. and Svyatskiya D. (2016). New preconditioning strategy for Jacobian-free solvers for variably saturated flows with Richards' equation. Adv. Wat. Res. DOI: 10.1016/j.advwatres.2016.04.016

[57] Ould Amy M., J.P. Magnan. (1965). Numerical modeling of flow and deformation in earth dams constructed on soft soils. Series "Studies and research LPC" series Geotechnics, No. 10. 145p. 1991

[58] Liakopoulos, A.C. Theoretical solution of unsteady unsaturated flow problem in soils. Bull. Intern. Assoc. Sci. Hydrology. Vol. 10, pp. 5-39.

[59] H. R. Thomas, Y. He, M. R. Sansom, C. L. W. Li. (1996). On the development of a model of the thermomechanical-hydraulic behavior of unsaturated soils, Engineering Geology, Volume 41, Issues 1-4, Pages $197-$ 218. https://DOI.org/10.1016/0013-7952(95)00033-X

[60] Benyelles Z. (1988). Modelling the flow of water into a partly saturated soil. Thesis of Master of Science by Research, 299 pages, University College, Cardiff; United Kingdom.

[61] Narasimhan, T.N. (1979). The significant of the storage parameter in saturated-unsaturated groundwater flow. Water Resources Res. 1-53 pp569-575. https:/DOI.org/10.1029/WR015i003p00569 